H I G H L I G H T S

GENE EXPRESSION

\section{Let's do the twist}

Specific gene-expression patterns define morphogenetic movements during development. Emmanuel Farge now reports, in Current Biology, that the reverse is also true - gene expression can be modulated by the mechanical strains of morphogenetic movements.

To study the effects of mechanical stress on gene regulation, Farge used micromanipulation to induce a unilateral $10 \%$ deformation for $5 \mathrm{~min}$ in the early Drosophila embryo and then tested the expression of early patterning genes. Expression of the dorsal-ventral gene Twist was induced around the dorsal-ventral axis, which resulted in the ventralization of the embryo.

Farge then showed that mechanical deformation caused the translocation of Armadillo to the nucleus. This dual-function protein - a transcription factor and a component of the cadherin adhesion complex at the plasma membrane - was a possible candidate for mediating Twist expression. Indeed, the expression of a dominant-negative form of dTCF/Pangolin (a co-factor necessary for Armadillo-dependent transcription) prevented expression of Twist in response to the shape change. So, Armadillo somehow mediates the mechanical induction of Twist expression.

When cells of the stomodeal primordium are compressed during the first phase of germ-band extension, which leads to anterior-gut formation, Twist expression increases eightfold. To test whether this effect is due to mechanical strain caused by germband extension, Farge analysed mutant embryos that did not undergo this morphogenetic process. The level of Twist expression in the mutants was similar to that before germ-band extension, but manual mechanical compression could rescue Twist expression in stomodeal cells.

Mutant embryos also had no Armadillo in the nucleus, but, again, this could be rescued by applying mechanical pressure to the stomodeal cells. So, the expression of Twist depends on the mechanically induced nuclear translocation of Armadillo.

The dorsal epithelium is normally subjected to pressure that is exerted by the extending germ-band at the posterior pole and that is transmitted to the anterior pole, thereby compressing the stomodeal cells against the invaginations of the mesoderm and foregut. Photoablation of the dorsal epithelium mechanically disconnects this tissue from the posterior pole and releases the compression of stomodeal cells, resulting in the lack of Twist induction. This finding confirms that Twist expression is mechanically induced by stomodealcell compression as a result of germband extension.

Farge concludes that, although the mechanism that triggers nuclear localization of Armadillo is not known, the fact that its homologue, $\beta$-catenin, translocates into the nuclei at the dorsal pole of early frog and fish embryos indicates that "...mechanical compression may reactivate a conserved and ancient pathway for dorsal-ventral axis formation" in the Drosophila embryo.

Arianne Heinrichs

\section{(2) References and links}

\section{ORIGINAL RESEARCH PAPER Farge, E.}

Mechanical induction of Twist in the Drosophila foregut/stomodeal primordium. Curr. Biol. 13, 1365-1377 (2003)

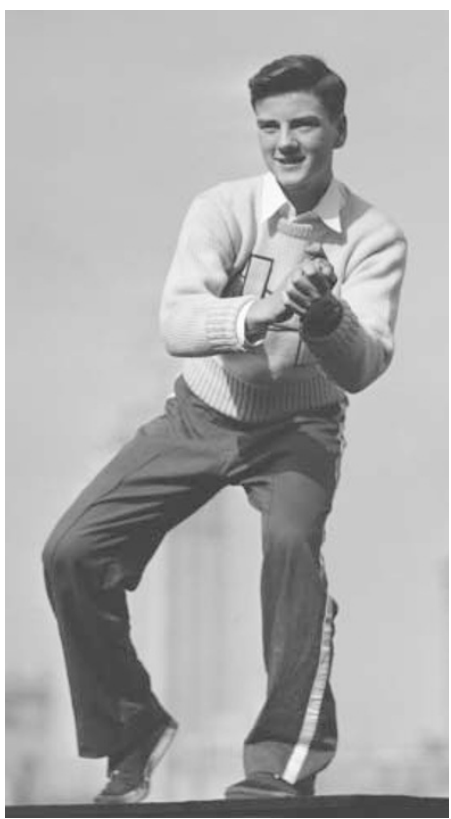

\section{STRUCTURE WATCH}

\section{Unravelling membrane transport}

Membrane transport proteins are crucial for maintaining a selective internal cellular environment. The largest subset of membrane transport proteins is the major facilitator superfamily (MFS), which converts electrochemical gradients into substrate concentration gradients, and transports ions, sugars, sugar-phosphates, drugs, amino acids and other hydrophilic solutes. In bacteria, MFS proteins are primarily responsible for nutrient uptake, although some act as drugefflux pumps and are involved in the development of antibiotic resistance.

Despite the importance of these proteins, there has been little progress in the determination of their structure and mechanism. However, two recent articles in Science now report the first high-resolution X-ray crystal structures of two MFS proteins from Escherichia coli: lactose permease (LacY), which catalyses the symport of lactose with $\mathrm{H}^{+}$; and the glycerol-3phosphate transporter (GlpT), which catalyses the exchange of glycerol-3-phosphate and inorganic phosphate. In the two papers, Abramson et al. report the structure of LacY in complex with the lactose homologue $\beta$-D-galactopyranosyl-1thio- $\beta$-D-galactopyranoside (TDG) to a resolution of $3.5 \AA$, and Huang et al. present the uncomplexed GlpT structure to 3.3- $\AA$ resolution.

Both LacY and GlpT are shaped like 'Mayan temples' and consist of two similar domains - the amino- and carboxyteminal domains - each of which contains six transmembrane helices. A central hydrophilic cavity that is open to the cytoplasm, yet closed to the periplasm, is also present in both structures, giving the overall structure a 'heart-shaped' topology when viewed normal to the membrane. The TDG molecule in the LacY structure is bound in this hydrophilic cavity, and is positioned in the centre of the membrane, almost equidistant from both the cytoplasm and periplasm consistent with the notion that MFS proteins have a single substrate-binding site. The high-resolution structures have identified several residues important for substrate binding.

To allow the transport of substrates across the membrane, there must also be a structural conformation in which the cavity is open to the periplasm. Based on the crystal structures, the authors of each paper propose a transport mechanism for MFS proteins, in which protonation and substrate binding induce several conformational changes. These conformational changes are postulated to result in a 'switching' of the opening of the hydrophilic cavity from the periplasm (outward-facing) to the cytoplasm (inward-facing), thereby allowing the transport of substrates across the membrane.

The authors have identified several residues from each structure as being important in facilitating the conformational changes necessary for the transition between the outward- and inward-facing conformations and, if a structure of the outward-facing conformation could be obtained, this mechanism, and the MFS story, would be complete.

Jane Saunders, Nature Reviews Microbiology

REFERENCES Abramson, J. et al. Structure and mechanism of the lactose permease of Escherichia coli. Science 301, 610-615 (2003) | Huang, Y. et al. Structure and mechanism of the glycerol-3-phosphate transporter from Escherichia coli. Science 301 , 616-620 (2003) 